\title{
Design of Semi-Adaptive 190-200 KHz Digital Band Pass Filters for SAR Applications
}

\author{
P Yadav \\ Dept of Electronics and \\ communication \\ SIST, Bhopal, India- 462031
}

\author{
A Khare \\ Dept of Electronics and \\ communication \\ SIST, Bhopal, India- 462031
}

\author{
K Parandham Gowd \\ Aisect University, Bhopal-Chiklod \\ Road \\ Raisen, Bhopal, India
}

\begin{abstract}
Technologies have advanced rapidly in the field of digital signal processing due to advances made in high speed, low cost digital integrated chips. These technologies have further stimulated ever increasing use of signal representation in digital form for purposes of transmission, measurement, control and storage. Design of digital filters especially adaptive or semi adaptive is the necessity of the hour for SAR applications. The aim of this research work is to design and performance evaluation of $380-400 \mathrm{KHz}$ Bartlett, Blackman and Chebyshev digital semi adaptive filters. For this work XILINX and MATLAB softwares were used for the design. As part of practical research work these designs were translated using FPGA hardware SPARTAN-3E kit. These were optimized, analyzed, compared and evaluated keeping the sampling frequency at $5 \mathrm{MHz}$ for 64 orders. Both these filters designed using software and hardware were tested by passing a sinusoidal test signal of $381 \mathrm{KHz}$ along with noise and the filtered output signals are presented.
\end{abstract}

Keywords-Digital filter; XILINX and MATLAB softwares; Field Programmable Gate Arrays (FPGA); SPARTAN-3E; DSP Chips.

\section{INTRODUCTION}

The extraordinary development in the field of high speed, low cost microelectronic digital IC's over the past few decades has stimulated an ever increasing use of signal representation in digital form for such purposes as transmission, measurement, storage and control. The conversion of a continuous signal to digital form makes possible the numerical manipulation of the data by IC's which is known as digital signal processing which is utmost necessary in the field of synthetic aperture radar applications(SAR). For remote sensing and detection applications digital signal processing concerns the techniques of processing the data to remove, for example, unwanted noise components, before the signal is reconstructed into analogue form, known as digital filtering, which is of interest in this research paper.

SAR data is required to be processed in real time, hence these filters are required to operate in real time. For real time operation, the complexity of the digital signal processing algorithm is limited by the condition that the numerical manipulation to determine each output sample must be performed in less than the sample period. Thus the design of a real-time filter generally involves a compromise between the complex requirements for a complex algorithm and a high sampling frequency.

The possibility of low-cost, real-time digital filtering first emerged in the 1970s when general purpose microcomputers were introduced. The early digital filters, however, had limited speed and precision, which restricted their use to lowfrequency applications and simple algorithms. In the 1980s, higher cost special purpose known as DSP chips were introduced, which contained much faster arithmetic units and on-chip memory for storing filter coefficients and data. In some cases, on chip A/D and D/A converters were included and subsequently rapid improvements in speed and complexity were made possible by ICs, thus resulting in design of digital filters of today. FPGA series of kits availability clearly helps researchers for taking up practical designs of semi adaptive band pass filters from lower to higher frequencies.

\section{Proposed Design Methodology}

\section{A. Softwares BaSed DESign.}

The simulated design methodology is as shown in fig.1. The design process involved the following steps:

1) MATLAB software tool is used to generate the coefficients required for the operation of the filter.

2) Xilinx software tool was used to design the filter.

3) Using this research methodology three types of filters namely, Bartlett, Blackman and Chebyshev Finite Interval Response (FIR) semi adaptive digital band pass filters for 190-200 KHz were designed.

4) Filter response was derived for the sampling frequency of $5 \mathrm{MHz}$ of order 64.

5) MATLAB software program was applied for calculating and plotting of SNR

The (FDA) Filter Design and Analysis tool in MATLAB provides the option to design the digital filter to offer the respective response and coefficients to be implemented within the design using VHDL. Filter design can be carried out often selecting various options available in FDA tool for generation of required coefficients for the respective filter from the target menu using the $\mathrm{C}$ header option of the FDA tool. The generated coefficients in the $\mathrm{C}$ header files are then used in the VHDL file for the digital filter designing which is to be convoluted with the sampled data of SAR. 


\section{B. HARDWARE BASED DESIGN}

The software based design methodology described above was translated on FPGA kit using SPARTA-3E for the practical experimental work. The inputs and outputs were taken on digital storage oscilloscope. In this research work the following practical circuit design steps was carried out as per methodology shown in Fig.2. The circuit designed using the SPARTAN Logic IC X-C3S, a seven segment display, DIP switches is as shown in Fig.3.

1) Using this FPGA kit three types of filters namely, Bartlett, Blackman and Chebyshev Finite Interval Response (FIR) semi adaptive digital band pass filters for $380-400 \mathrm{KHz}$ were practically designed.

2) The SPARTAN Logic IC contains 4X10-6 logic gates and has 208 pins.

3) 8 DIP switches were incorporated for selecting various combinations such as frequency of the filter, sampling rates and input signal.

4) 16 bit $A / D$ and D/A converters are used for conversion and reconstruction of samples.

5) This filter was integrated into the circuit testing system as shown in fig.4.

6) Here a low power test signal (-5dB) of $195 \mathrm{KHz}$ was mixed with AWGN noise and this mixed signal was passed through designed band filters namely Bartlett, Blackman and Chebyshev for the frequency $190-200 \mathrm{KHz}$.

In the hardware set up out of 8 DIP switches two are used for input/output, two are for selecting sampling rate and four switches for selecting filter coefficients. These four switches with 16 bit data it is possible to generate 64 sets of filter coefficients.

This technique of using Field Programmable Gate Array with DIP switches as shown in Fig.4 for selecting sampling rate for noise, test signal and various filter coefficients can be termed as a concept of semi-adaptive filter design. With this semi-adaptive technique depending on the test signal and noise condition particular set can be recalled there by increasing the signal to noise ratio for SAR to enable better detection. Here simulated and generated SAR signals accompanied with random noise is sampled at a frequency of $5 \mathrm{MHz}$.

Here SPARTAN-3E was used, configured in designing the 3 types of digital filters in this paper. The achieved filter circuit is as shown in figure.3.
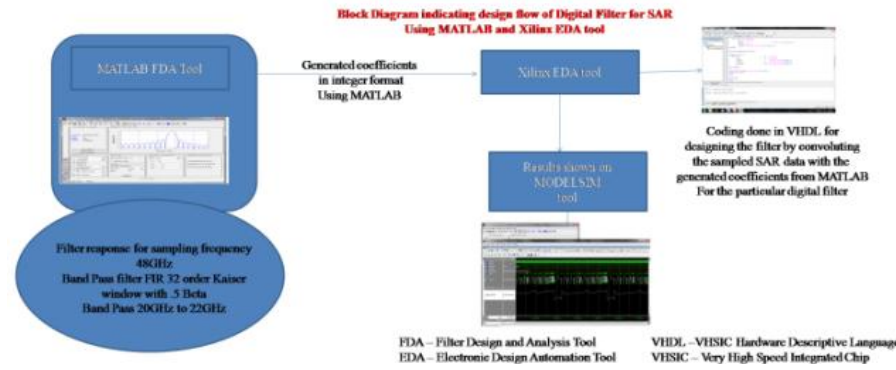

Fig.1. Block diagram of the Design using software methodology

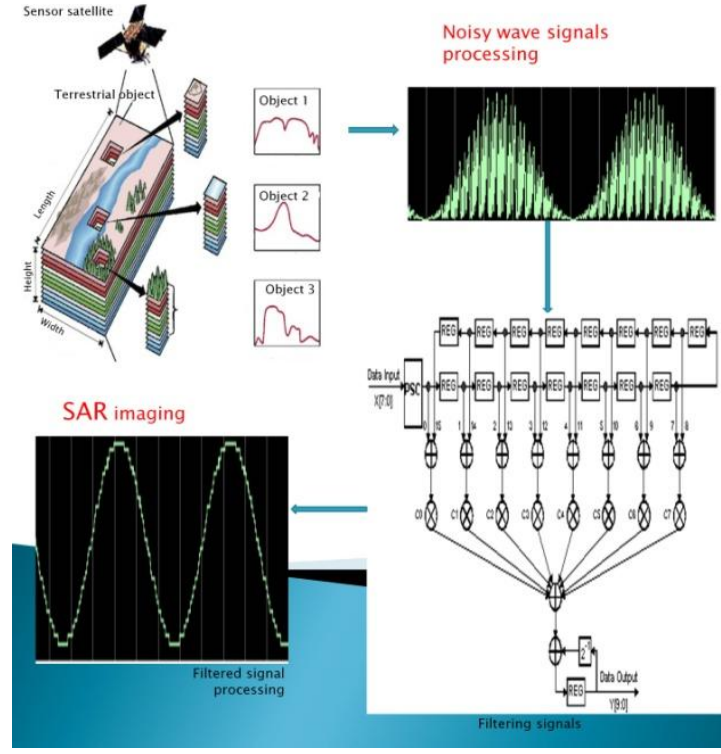

Fig.2. Block Diagram of the Design using Hardware methodology.

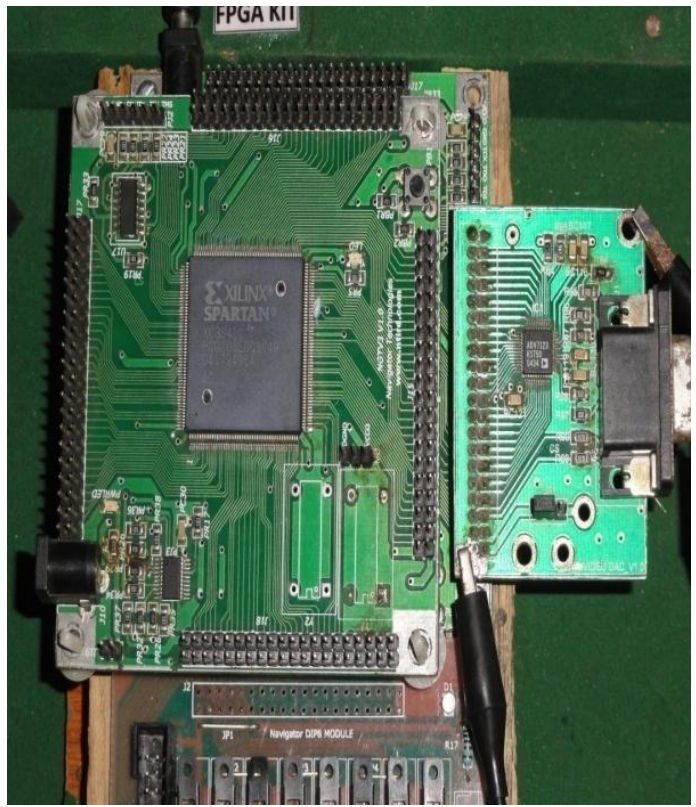

Fig.3. Designed circuit using FPGA kit.

\section{Results AND Discussion}

Block diagrams used in these designs by software and hardware are as shown in figures 1 and 2 respectively. The designed circuit and integrated experimental setup utilized for this research work are depicted in fig. 3 and 4 respectively.

The frequency responses of Bartlett, Blackman and Chebyshev FIR band pass filters of order 64 for a sampling frequency of $5 \mathrm{MHz}$ are as shown in figures 5, 6 and 7 respectively. The original sinusoidal test signal of $195 \mathrm{KHz}$ was passed through this 190 to $200 \mathrm{KHz}$ digital band pass FIR filters of Bartlett, Blackman and Chebyshev of order 64 which was designed using XILINX and MATLAB soft wares are as shown in Figures 8, 9 and 10.respectively. The figures 8, 9 and also show the SNR improvement versus iteration number 
calculated and plotted for Bartlett, Blackman and Chebyshev types of filters. Figure 11 shows the test signal,test signal with noise and filterd signals obtained from practical experimental set up using FPGA kit (SPARTAN-3E kit) observed in a two channel digital oscilloscope.

It is clearly observed from figure 8 that Bartlett type of digital FIR band pass filter clearly suppresses the noise and the output signals passed with and without noise are exactly comparable. The observations from figures 9 the Blackman filter and 10 the Chebyshev filter indicate that noise is not suppressed completely and output is seen with some noise components and far inferior to Bartlett type of filter of the same design.

From this figures 8 and 11, both are of Bartlett types designed using software and hardware respectively, it is clearly seen that noise is suppressed and the test signal has been passed. The figure 11 shows the input test signal and filtered output signal which was passed through the same filter which was designed using SPARTA 3 E kit with logic gates observed in a two channel digital storage oscilloscope. The simulated filter and the practically designed filter have rejected the noise and are comparable with hardly any differences as per expected lines.

It is seen from the figures 9, the Blackman and 10, the Chebyshev types of filters of software based design, that the original signal is completely mixed with noise indicating inferiority of these filters when compared with Bartlett filter of same design.

It is seen the fig. 3 and 4 that the band pass filter design using SPARTA 3E Kit was clearly translated and achieved practically using the methodology proposed in this paper. It is seen from the figures. 8 and 10 that the simulated SAR signal clearly passing through the 190 to $200 \mathrm{KHz}$ window in software based and practically designed band pass filters, by suppressing the noise levels, presenting the reflected echo of the SAR signal.

From figures 8, 9 and 10 which also shows the SNR improvement factor versus iteration number, it is clearly observed that SNR improvement factors achieved for Bartlett type of filter is $7.78 \mathrm{~dB}$, for Blackman type of filter is $7.692 \mathrm{~dB}$ and for Chebyshev type of filter is $7.42 \mathrm{~dB}$. Dominance of Bartlett type filter is very clear from the same design achieved for SNR improvement.

\section{CONCLUSION}

In this research work the XILINX and MATLAB softwares based Bartlett, Blackman and Chebyshev FIR digital 190-200 $\mathrm{KHz}$ semi adaptive band pass filters design methodology was successfully translated in to practical one using SPARTAN-3E kit along with $\mathrm{A} / \mathrm{D}, \mathrm{D} / \mathrm{A}$ converters and DIP switches and seven segment display for 64 orders for a sampling frequency of $5 \mathrm{MHz}$.

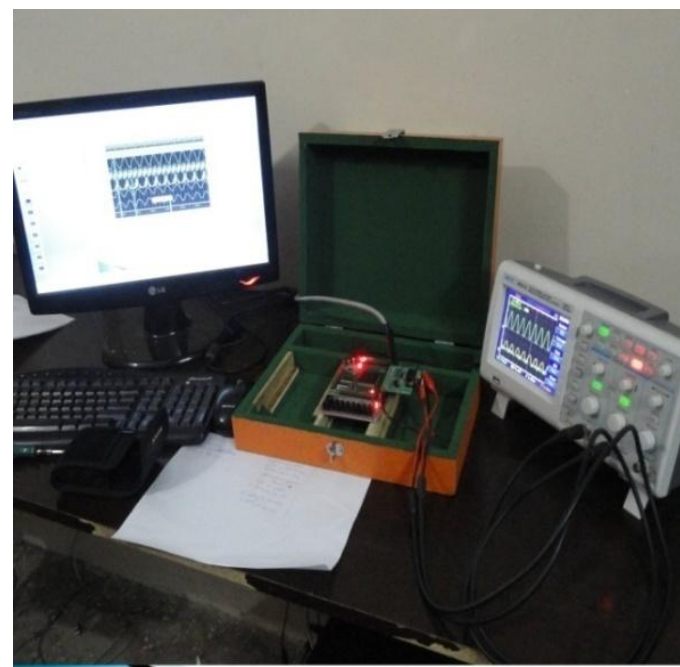

Fig.4. Designed circuit of the proposed methodology.

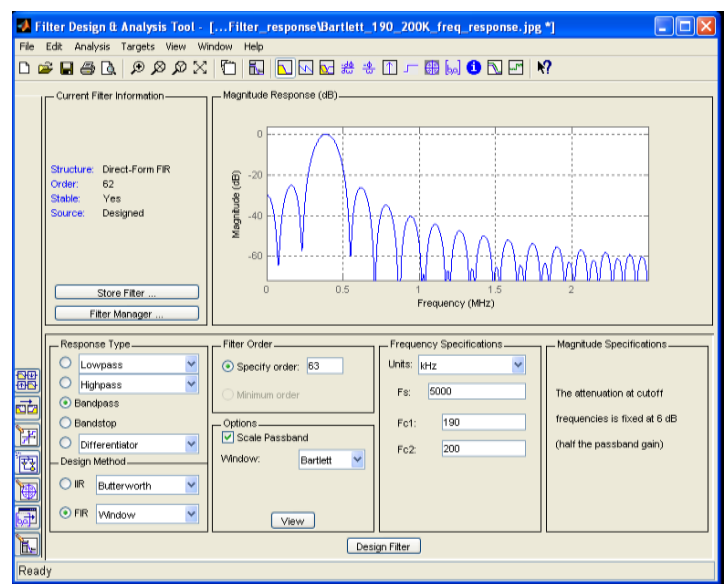

Fig.5. Frequency Response (Bartlett)

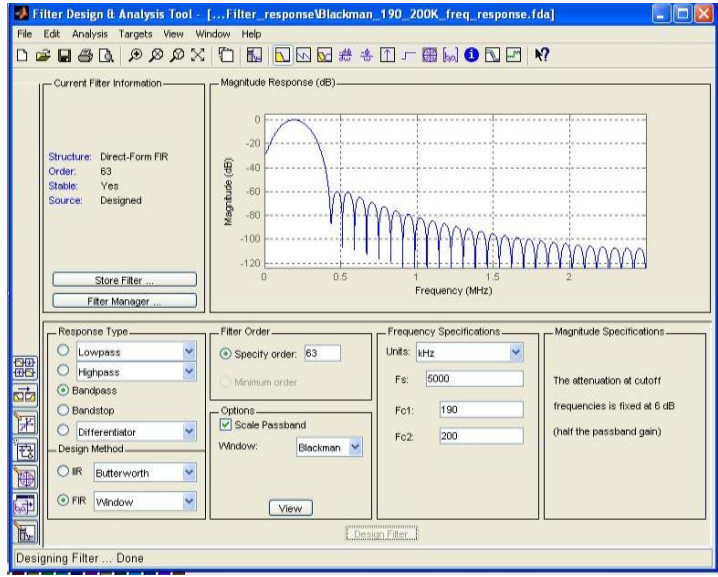

Fig.6. Frequency Response (Blackman) 


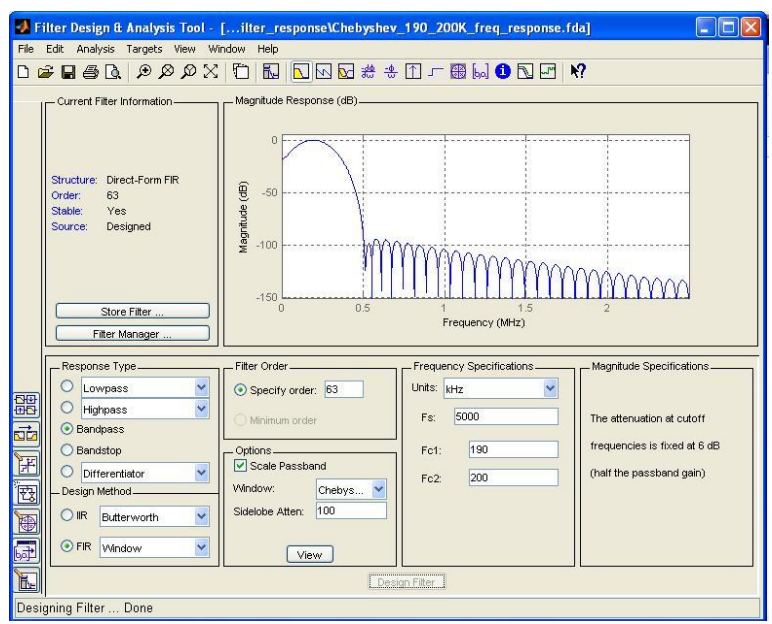

Fig.7. Frequency Response (Chebyshev)

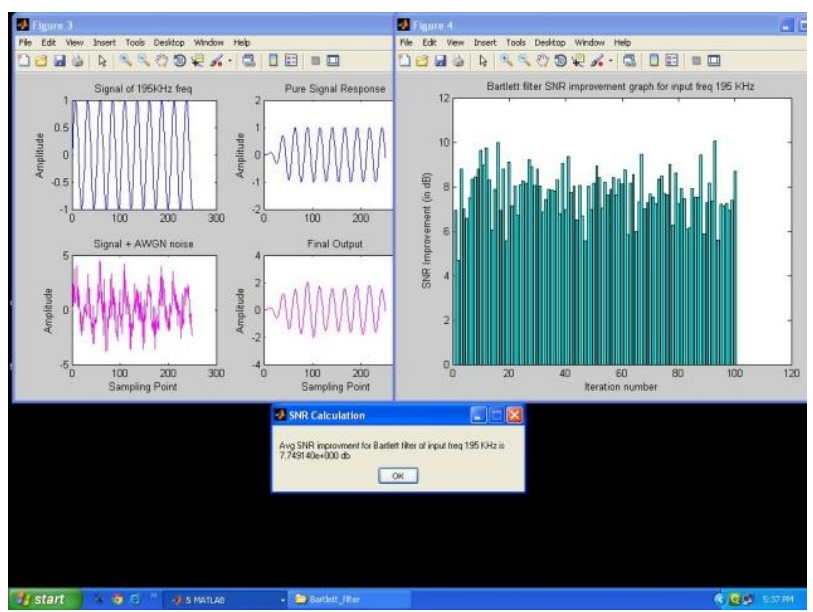

Fig.8. Input signal, signal+ noise, outcomes and SNR improvement (Bartlett)

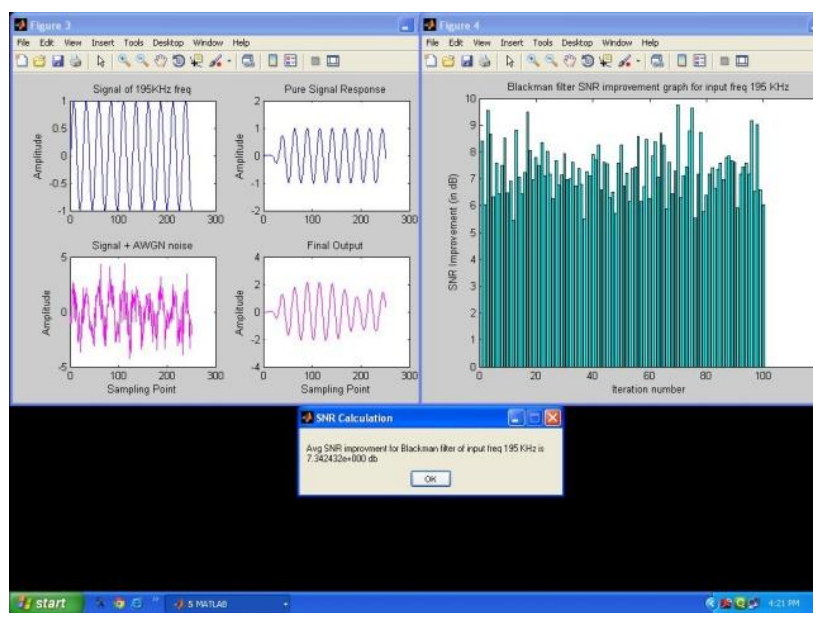

Fig.9. . Input signal, signal+ noise, outcomes and SNR improvement (Blackman)

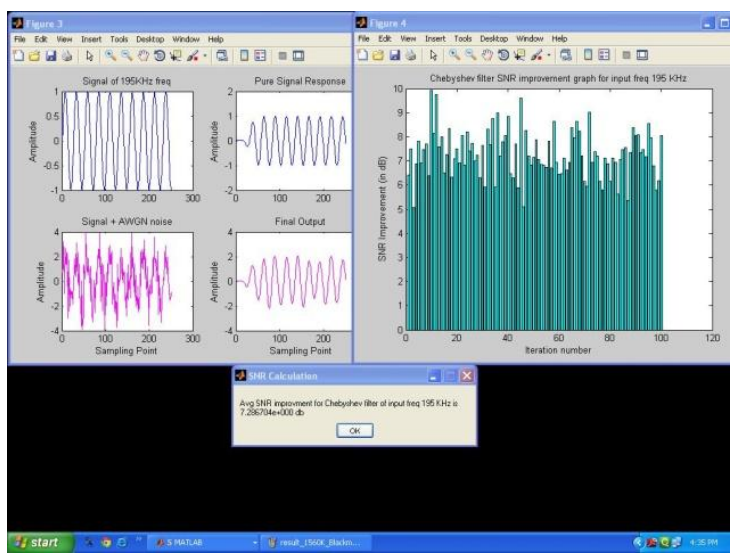

Fig.10. Input signal, signal+ noise, outcomes and SNR improvement (Chebyshev)

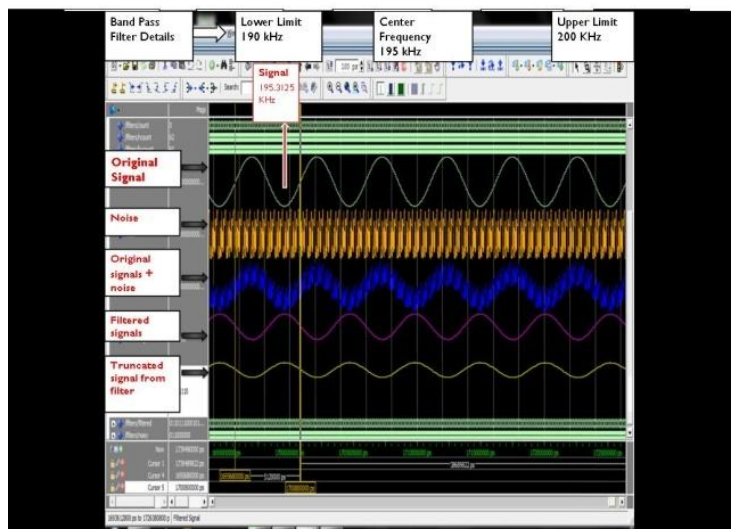

Fig.11. Figure 11. Original signal, noise + signal and filtered signals

The response was successfully obtained for the designed frequency of $195 \mathrm{KHz}$ from both the filters theoretical and practical. The superiority performance of Bartlett type of filter is clearly observed from both types of design using software tools and FPGA kit practically over Blackman and Chebyshev type of filters from the same designs.

SNR improvement factor of $7.78 \mathrm{~dB}$ achieved for Bartlett is higher than Chebyshev of $7.42 \mathrm{~dB}$ and Blackman of 7.692 $\mathrm{dB}$ conforming that better quality low frequency FIR semi adaptive digital band pass filters can be achieved through Bartlett type. These filters are suitable for low frequency SAR applications to mitigate random noise levels and give the desired target response so that resolution and identification of desired objects are achieved.

The SPARTAN-3E digital signal processing chip was successfully configured, designed and achieved for semiadaptive digital FIR filters and test signal was passed with noise and output clearly obtained as shown in figure 11 especially for Bartlett type. 
Filters designed in this paper analyzed and evaluated which are comparable and matching well in their responses of filtering out unwanted noise and passing the original signal in the designed frequency band.

In this paper a concept of low frequency digital filters for synthetic aperture radar applications for the purpose of deep detection on earth surfaces and deep sea bed are suitably demonstrated. High frequency SAR images are deteriorated by speckle noise, so in this paper an attempt is made to design a practical digital filter using SPARTA 3E kit for low frequency SAR applications. It is well known that lower frequencies have longer wave length and hence can penetrate deeper in to earth and sea bed for detection applications of SAR.

\section{Future SCOPE}

This experiment on practical circuit design of semiadaptive digital filters using SPARTAN-3E and software based design using XILINX and Mat Lab softwares for SAR applications shows the possibility to formulate fully adaptive digital filters for Remote Sensing applications such as for Disaster management, mining, Forest management and military applications. These filters will play a crucial role in practical implementation in future SAR noise reduction.

\section{REFERENCES}

[1] Tomlinson G, H, "Electrical Networks and Filters (Theory and Design)", Prantice Hall Internationalo (UK) Ltd, 1991.

[2] Rhodes, J.D, “Theory of Electrical Filters", London, John Wiley,1976.

[3] Van Valkenberg, M,E, “Analog Filter Design”, London, Holt Rinehart and Winston, 1987.

[4] D.M Pozar and D.H Schaubert "The Analysis and Design Of Microstrip Antenna Array".

[5] K. Ragopal, J. Dinesh Babus, Venkataraman, Generalized adaptive IFIR Filter Bank structures, Science Direct Signal Processing, pp 1-20, 2007.

[6] Skolnik, M. I., Radar Handbook, McGraw-Hill, New York, 1970.

[7] Curlander, J. C. and R. N. McDounough, Synthetic Aperture Radar, Systems and Signal Processing, John Wiley \& Sons, New York, 1991.

[8] Ulaby, F. T., R. K. Moore, and A. K. Fung, Microwave Remote Sensing: Active and Passive, Vol. I, Artech House, Norwood, 1981.

[9] Drinkwater, M. K., R. Kwok, and E. Rignot, "Synthetic aperture radar polarimetry of sea ice," Proceeding of the 1990 International Geoscience and Remote Sensing Symposium, Vol. 2, 1525-1528, 1990.
[10] Lynne, G. L. and G. R. Taylor, "Geological assessment of SIRB imagery of the amadeus basin," IEEE Trans on Geosc. And Remote Sensing, Vol. 24, No. 4, 575581,1986

[11] Hovland, H. A., J. A. Johannessen, and G. Digranes, "Slick detection in SAR images," Proceeding of the 1994 International Geoscience and Remote Sensing Symposium, 2038-2040, 1994.

[12] Walker, B., G. Sander, M. Thompson, B. Burns, R. Fellerhoff, and D. Dubbert, "A high-resolution, four-band SAR Testbed with real-time image formation," Proceeding of the 1986 International Geoscience and Remote Sensing Symposium, 1881-1885, 1996.

[13] Storvold, R., E. Malnes, Y. Larsen, K. A. Hogda, S.-E. Hamran, K. Mueller, and $\mathrm{K}$. Langley, "SAR remote sensing of snow parameters in norwegian areas Current status and future perspective," Journal of Electromagnetic Waves and Applications, Vol. 20, No. 13, 1751-1759, 2006.

[14] Kong, J. A., S. H. Yueh, H. H. Lim, R. T. Shin, and J. J. van Zyl, “Classification of earth terrain using polarimetric synthetic aperture radar images," Progress In Electromagnetics Research, PIER 03, 327-370, 1990.

[15] Thompson, T. W., A User's Guide for the NASA/JPL Synthetic Aperture Radar and the NASA/JPL L- and C-band Scatterometers, 83-38, JPL Publication, 1986.

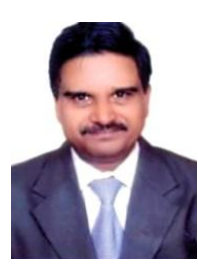

AUTHOR'S INFORMATION

Prof. P Yadav is a post graduate from IIT- Kanpur (India) and a research scholar in Electronics Engg. He has served in Indian Air Force as commissioned officer for 25 yrs, and nine yrs, as a technocrat academician in reputed Engg. Institutes. He is an entrepreneur who has many project patents under his name. He is a fellow of IETE.

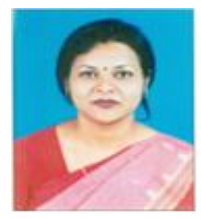

Dr Anubhuti Khare received her BE in Electronics and Communication from Government Engineering College, Bhopal in 1994. She obtained her M.Tech and Ph D in Electronics and Communication from MANIT, Bhopal. Presently she is working as Associate Professor in E \& C Department, UIT, RGPV, Bhopal. She has more than 50 publications to her credit.

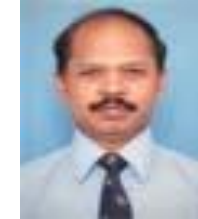

K Prandham Gowd obtained his B.Tech in Electronics and Communication Engineering with distinction from S.V. University, Tirupati (India) and ME (Microwaves and Radar) from IIT Roorkee. In 1994 he has conducted RCS Reduction experiments on coated (by pasting of absorber sheets) and uncoated scaled models of aircraft which is first time in India at IIT Roorkee. He has 41 research publications and 05 Technical reports to his credit most of them on RCS/RCS Reduction/Stealth Technology. He has one copyright to his credit on Dynamic RCS Range Validation Procedure from Govt of India. He is a Life Member of All India Management Association (AIMA), AeSI and Fellow of IETE. He had authored a book on Stealth Aircraft Technology. 